task, divided into four main parts-(1) "The Physical World"; (2) "The Realm of Life"; (3) "Mentality"; (4) "Outline of a Metaphysic". These headings are enough to show the scope which the book is intended to embrace. It is very readable, even though there is a fair proportion of paste and scissors, with a slight tendency to write round the references. In dealing with the question of intelligence, a warning is given of the effect of drawing a sharp line between logic and psychology, which is so marked a feature of recent discussion. This is the result of a determ. ination to avoid psychologism, the pet aversion of the phenomenologists and the Husserl school. The principles of organization are eliminated, and with them purposeful thought. Broadly, the Gestalt concept bears up well: it has seen many modifications since the days of Wertheimer and Koffka, but its integrative features are still valuable, much more indeed than mere 'and-summations'. Mozart (quoted by the author) would survey a musical composition "all at once", the whole being instantly established.

The unique position of entropy, to which reference has already been made, appears as the connecting link not only between metaphysics and science but also between our conception of the living and the non-living, which is roughly that of its dominance in open and closed systems respectively.

The wonder is that the living organism is so constructed as to be a means whereby entropy can not only be held constant but also in some cases even decreased. This is what the communications engineer strives to do by making use of negative entropy, $K \log \mathrm{l} / D$, wherein $D$ is a measure of noise. Apart from the mathematical formalism, we begin to see in organization a signpost to a better understanding of meaning and perception. Naturally, once this connexion between entropy and living activity is realized, there is scarcely any limit beyond which it is not applicable.

Prof. Harris makes the point that modern symbolic logic, with its great capacity for preventing mistakes, adds no contribution to the pressing need for a science which treats of what organization is supposed to do, namely to set up the forms and principles of thinking. Ethically, as the author says, it is only at the heights of human effort that, due to such vast complexity, aberrations can occur at all. It is still true that man tends to kill the object he loves.

The thesis which these two books are written to defend - essentially a study of contrasts-is best summed up metaphorically in the words which "My Lady Philosophy" used to console Boetius in his prison cell at Pavia in A.D. 524. She said to him, "I will show thee the way which shall carry thee home", which is good and useful science. But she added, "I will give thy mind wings to be raised aloft", which is metaphysics.

F. I. G. RAwLINS

\section{TEACHING RELATIVITY}

Introduction to General Relativity

By R. Adler, M. Bazin and M. Schiffer. (International Series in Pure and Applied Physics.) Pp. xv +451. (New York, Toronto, London and Sydney: McGraw-Hill Book Company, 1965.) 100s.; 12.50 dollars.

YENERAL relativity has become a popular and widely J understood subject in the past 20 years. As a result there have been a large number of books on the subject, some of them presenting collections of original work, and others presenting a view of the whole subject from one particular point of view. The present book differs from all recent books (except perhaps that of Synge) in being a genuine text-book. It is based on a course of lectures, given at Stanford University, by Schiffer. The aim of the authors was both to direct the attention of mathematicians to a fruitful field of research and simultaneously to provide the physicists with a simple introduction into the powerful mathematical methods used in general relativity.
Great care is taken to explain the motivation for everything that is being done, and the whole theory is set out with great clarity. After an introduction dealing with non-Euclidean geometry the tensor calculus is set up. The notation is the classical one with suffixes, no attempt being made to bring in the exterior differential calculus. The relationship of tensor calculus to physics is introduced by means of Maxwell's equations in a flat space and also through a discussion of gravitational red shifts in terms of a description of gravitation by means of a curved space. This brings the reader up to the position of having "general relativity without field equations". The usual arguments are now brought forward for the Einstein field equations as generalizations of Laplace's equation. Here, however, the authors do not neglect to use the argument from the equation of geodesic variation which exhibits the connexion with Laplace's equation in its clearest form.

Having now formulated the theory, discussion on the spherically symmetric static solution and the linearized. field equations follow. After the derivation of the Schwarzschild solution there is a very careful description indeed of the present position of the theory with regard to the three so-called 'crucial tests'. A particularly interesting chapter follows, on the mathematical structure of the field equations in relation to the initial value problem. The characteristics and the bi-characteristics of the equations are exhibited with great clarity. The authors elect to omit the general (Fourès) proof of the existence and uniqueness, but they do give the particular result of Lichnerowicz (that a static empty universe which is flat at infinity is flat everywhere). It will give an idea of the completeness of the treatment to mention that this chapter concludes with a proof of the maximum principle for the generalized Laplace equation (that is, Earnshaw's theorem in curved space).

There are also chapters on conservation laws, cosmology and electromagnetism (including Weyl's theory and the already-unified theory of Rainich and Wheeler). The book is beautifully printed and has an excellent index.

C. W. Kulmister

\section{THE SUN}

\section{A Star Called the Sun}

By Dr. George Gamow. Pp. xiii +208. (London: Macmillan and Co., Ltd., 1965.) 35s. net.

$\mathrm{D}$ R. GAMOW has written an entirely new book on the subject of his earlier work "The Birth and Death of the Sun", and has incorporated an account of the advances in solar research which have occurred in the interim.

The book begins with a survey of man's attempts through the ages to answer such questions as how far the Sun is from the Earth, how large it is and how hot. Then follow chapters on the solar spectrum and what it tells us about the composition of the Sun, the turbulent solar surface, its atmosphere and its corona, and its hot interior. We are reminded of Dante on his journey to the inferior regions: "I would have cast me into molten glass to cool me, when I enter'd, so intense raged the conflagrant mass". After analysing the sources of stellar energy at different stages of the life of a star, the author compares the Sun with other stars and explores the nature of such old ones as red giants, white dwarfs and supernovae, the stars that "die glamorously". The last section of the book, on the origin of stellar families, shows that at least one billion stars in the Milky Way may possess planetary systems like that of our Sun (an American billion is a thousand million). Dr. Gamow is an expert in this branch of astrophysics and, as is expected, the sections that deal with the evolution and decay of stars are accurate, written with conviction, and fascinating.

Some of his statements concerning fundamental physics are not always so happy. For example, the 'decay period' [sic] of radium is given as 1,840 years, and two pages 